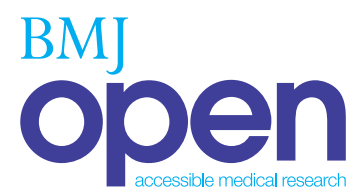

\title{
Do statins interfere with lifestyle intervention in the prevention of diabetes in primary healthcare? One-year follow-up of the FIN-D2D project
}

To cite: Rautio N Jokelainen $\mathrm{J}, \mathrm{Oksa} \mathrm{H}$, et al. Do statins interfere with lifestyle intervention in the prevention of diabetes in primary healthcare? One-year follow-up of the FIN-D2D project. BMJ Open 2012;2: e001472. doi:10.1136/ bmjopen-2012-001472

- Prepublication history and additional material for this paper are available online. To view these files please visit the journal online (http:// dx.doi.org/10.1136/bmjopen2012-001472)

Received 15 May 2012 Accepted 15 August 2012

This final article is available for use under the terms of the Creative Commons Attribution Non-Commercial 2.0 Licence; see http://bmjopen.bmj.com

For numbered affiliations see end of article.

Correspondence to Dr Nina Rautio; nina.rautio@oulu.fi

\section{ABSTRACT}

Objectives: To examine whether the use of statins is associated with the incidence of type 2 diabetes (T2D) and changes in glucose metabolism among individuals at high risk for T2D participating in 1-year lifestyle intervention in primary healthcare setting.

Design: Prospective follow-up study.

Setting: In all, 400 primary healthcare centres and occupational healthcare clinics in Finland.

Participants: We screened altogether 10149 individuals at increased risk for T2D; of these, 2798 non-diabetic individuals verified by a $2 \mathrm{~h}$ glucose tolerance test participated in the 1-year follow-up. Interventions: Lifestyle intervention (individual and/or group-based counselling).

Primary outcome measures: Incidence of $\mathrm{T} 2 \mathrm{D}$ and fasting and $2 \mathrm{~h}$ glucose measured at baseline and follow-up.

Results: A total of 484 individuals (17.3\%) used statins at the baseline. Of them $31(7.5 \%)$ developed T2D during the follow-up, compared to $126(6.5 \%)$ of statin non-users (OR $1.17,95 \% \mathrm{Cl} 0.78$ to $1.76, \mathrm{p}=0.442$ ). Interestingly, fasting glucose increased by $0.08 \mathrm{mmo} / / \mathrm{l}$ in statin users, but remained unchanged in non-users, the difference being $0.074 \mathrm{mmol} / \mathrm{l}(95 \% \mathrm{Cl} 0.014$ to 0.134$)$ and remained significant even after adjustment for age, sex, baseline fasting glucose, the presence of cardiovascular disease (CVD), use of antihypertensive and/or coronary artery disease medication, weight and 1 -year weight change (adjusted $p=0.042$ ).

Conclusions: The incidence of T2D did not differ between the statin users and non-users. The finding that fasting glucose slightly increased in statin users in spite of lifestyle interventions suggests the view that the use of statins might have unfavourable effects on glucose metabolism and that statins might hamper beneficial effects of lifestyle intervention in people at high risk of T2D.

\section{INTRODUCTION}

Statin therapy is effective in the primary and secondary prevention of cardiovascular diseases (CVD) ${ }^{1-3}$ Recently, a slightly increased

\section{ARTICLE SUMMARY}

Article focus

- Statin therapy has been suggested to slightly increase the risk of type 2 diabetes (T2D). It is not known whether this increased risk could be counteracted by lifestyle intervention.

- In this paper, we report the results of the analyses of use of statins and their association to the incidence of T2D and changes in glucose metabolism among individuals at high risk for T2D participating in lifestyle intervention in primary healthcare.

Key messages

- The incidence of T2D did not differ between the statin users and non-users. Fasting glucose increased in statin users compared to non-users and remained significant even after adjustment for sex, age, baseline fasting glucose, the presence of cardiovascular disease (CVD), use of antihypertensive and/or coronary artery disease medication, weight and 1-year weight change.

- The finding that fasting glucose slightly increased in statin users in spite of lifestyle interventions suggests that the use of statins might have unfavourable effects on glucose metabolism and that statins might hamper beneficial effects of lifestyle intervention in people at high risk of T2D.

Strengths and limitations of this study

- Implementation project of the national diabetes prevention programme in Finland. (FIN-D2D) is the first national effort to implement the prevention of diabetes in a primary healthcare setting and as far as we know this is the first study to examine the association of use of statins and T2D risk in high-risk individuals for T2D who participated in the lifestyle intervention programme.

- High number of individuals who were screened at baseline did not participate in the 1-year follow-up examination.

- The use of statins was based on self-report. Because of the observational data, we cannot elicit a causal relationship. 
type 2 diabetes (T2D) risk has been linked to the use of statins, ${ }^{4-7}$ although the West of Scotland Coronary Prevention Study (WOSCOPS) showed that statin users had a $30 \%$ reduction of the hazard of becoming diabetic. ${ }^{8}$ However, recent meta-analyses show that the risk of T2D is 9-13\% higher in statin users compared to non-users, ${ }^{4} 7$ and the effect has been shown to be dose dependent, ${ }^{9}$ and age dependent. ${ }^{7}$ T2D is shown to be prevented or delayed by lifestyle counselling aimed at weight reduction, healthy diet and increase in physical activity in controlled lifestyle intervention trials. ${ }^{10-14}$ We recently carried out a 1-year lifestyle intervention programme, FIN-D2D, in primary healthcare settings in Finland to find out whether lifestyle interventions work in real life as well. ${ }^{15}$ This study also included a reasonable number of statin users and gave us an impetus to study whether statin therapy had associations with the incidence of T2D and changes in glucose metabolism during the 1-year follow-up among individuals at high risk for T2D participating in lifestyle intervention in primary healthcare.

\section{MATERIAL AND METHODS}

This study belongs to the implementation project of the Programme for the Prevention of T2D in Finland 2003-2008 (FIN-D2D). ${ }^{16}{ }^{17}$ In total, 400 primary healthcare centres and occupational healthcare outpatient clinics participated in the programme. ${ }^{15}$ One of the principal aims of the FIN-D2D was to screen for individuals at high risk of T2D,${ }^{16}$ by using a modified Finnish Diabetes Risk Score test (FINDRISC), ${ }^{15} 1819$ with a cut-off point of 15 or more and history of impaired fasting glucose (IFG), impaired glucose tolerance (IGT), cardiovascular events or gestational diabetes. ${ }^{15}$ Lifestyle counselling was offered to high-risk individuals in order to reduce their T2D risk. There was no randomisation, instead an opportunistic screening process was conducted and participants volunteered, thus they provided oral consent for willingness of taking part in this national programme carried out within primary care to prevent diabetes in the normal clinical settings. Because from the participants' point of view, they were not participating in a 'study'; there was no need to ask for a separate written informed consent from them. Nevertheless, all participants received written information on the FIN-D2D and they were informed of the data collection. As for the ethical approval, the National Public Health Institute applied for and obtained the permission from the Ministry of Social Affairs and Health for the purposes of evaluation and research to collect register-based morbidity data on FIN-D2D participants from primary care information systems.

Lifestyle counselling was performed either in group sessions or individually. Local resources had an effect on the number and frequency of intervention visits. However, individual visits were planned according to individuals' needs, and the following topics and items were included: weight control, meal frequency and quality, for example, use of dietary fibre, fat and salt, physical activity, smoking, alcohol use and diabetes as a disease in general. Group sessions were mainly weight maintenance or exercise groups and lectures concerning diabetes and lifestyle changes. ${ }^{15} 17$

After identification individuals at high risk for T2D were referred to health check-ups. They were asked to fill in a questionnaire on background health and lifestyle information before the health check-up. Local nurses in primary healthcare measured weight (to the nearest $0.1 \mathrm{~kg}$ ), height (to the nearest $\mathrm{cm}$ ), systolic and diastolic blood pressure (BP) ( $\mathrm{mm} \mathrm{Hg}$ ) and gave brief motivationbuilding and counselling for lifestyle changes, ${ }^{17}$ based on the principles of the Finnish Diabetes Prevention Study (DPS). ${ }^{20}$ Body mass index (BMI) was calculated by weight divided by height $\left(\mathrm{kg} / \mathrm{m}^{2}\right) .{ }^{15}$ Weight loss during follow-up was dichotomised by using median to $\leq 0.80$ or $>0.80 \mathrm{~kg}$ for both genders. Oral glucose tolerance test (OGTT) was carried out in the morning after overnight fast with a glucose load of $75 \mathrm{~g}$. Fasting and $2 \mathrm{~h}$ samples were collected from venous or capillary blood samples. At the baseline $80 \%$ and at the follow-up $85 \%$ were plasma venous samples. Corresponding figures for capillary samples were $20 \%$ and $15 \%$, respectively. ${ }^{15}$ Glucose tolerance status was classified by using World Health Organization (WHO) 1999 criteria. $^{21}$ Enzymatic methods were performed locally to determine plasma lipids and lipoproteins $(\mathrm{mmol} / \mathrm{l}){ }^{15}$

The use of lipid-lowering medication, antihypertensive and coronary artery disease medication was verified at the health check-up at baseline from prescriptions if possible. Individuals whose lipid-lowering medication had been initiated 120 days before the first visit or before the midway of the 1-year follow-up (no later than 270 days after the first visit) were regarded as users at the baseline. Statins are used almost exclusively for the treatment of dyslipidaemias in Finland. ${ }^{22}$ In 2010, 93035 individuals had reimbursement of drug costs under the Special Refund Category for dyslipidaemia associated with chronic coronary artery disease for HMG-CoA reductase inhibitors, while the corresponding numbers for fibrates and bile acid sequestrates were only 573 and 104, respectively. ${ }^{23}$ Thus, we had a good reason to assume that lipid-lowering medication included statins only. If an individual used antihypertensive or coronary artery disease medication or both at the baseline he/she was classified as a user. CVD (heart failure, angina pectoris, coronary artery disease, myocardial infarction, heart bypass surgery and angioplasty) were asked by the questionnaire. If an individual had one of these diseases or abnormalities, he/she was considered as having CVD.

In total, 10149 (3379 men and 6770 women) high-risk individuals aged 18-87 years were contacted between 17 January 2004 and 28 August 2007; 8353 of them had OGTT. Of them 5523 individuals participated in the follow-up visits, and 1-year follow-up information (defined by visits between 17 January 2005 and 12 June 2008) was available for 3880 individuals (1339 men and 
Table 1 Cardio-metabolic risk factors at the baseline according to use of statins in a high-risk cohort of the FIN-D2D

\begin{tabular}{|c|c|c|c|c|c|}
\hline \multirow[b]{3}{*}{ Variable } & \multicolumn{4}{|c|}{ Use of statins } & \multirow[b]{3}{*}{ p Value } \\
\hline & \multicolumn{2}{|c|}{ User } & \multicolumn{2}{|c|}{ Non-user } & \\
\hline & $\mathbf{N}$ & Mean (SD) & $\mathbf{N}$ & Mean (SD) & \\
\hline Age (years) & 484 & $59.0(8.59)$ & 2314 & $53.7(10.6)$ & $<0.001$ \\
\hline Weight (kg) & 484 & $87.2(14.9)$ & 2314 & $87.8(16.9)$ & 0.442 \\
\hline BMI $\left(\mathrm{kg} / \mathrm{m}^{2}\right)$ & 484 & $31.0(4.58)$ & 2302 & $31.5(5.30)$ & 0.027 \\
\hline Systolic BP (mm Hg) & 472 & $140(16.5)$ & 2276 & 139 (17.5) & 0.166 \\
\hline Diastolic BP (mm Hg) & 472 & 85 (9.00) & 2276 & $86(9.45)$ & 0.002 \\
\hline Total cholesterol (mmol/l) & 429 & $4.78(0.99)$ & 2051 & $5.26(0.95)$ & $<0.001$ \\
\hline HDL cholesterol (mmol/l) & 425 & $1.38(0.37)$ & 2028 & $1.43(0.43)$ & $0.019^{*}$ \\
\hline LDL cholesterol (mmol/l) & 415 & $2.65(0.87)$ & 1979 & $3.14(0.84)$ & $<0.001$ \\
\hline Triglycerides $(\mathrm{mmol} / \mathrm{l})$ & 424 & $1.68(0.90)$ & 2019 & $1.56(0.95)$ & $0.001^{*}$ \\
\hline Fasting glucose (mmol/l) & 424 & $5.84(0.55)$ & 2008 & $5.77(0.58)$ & 0.016 \\
\hline $2 \mathrm{~h}$ glucose $(\mathrm{mmol} / \mathrm{l})$ & 421 & $7.11(1.81)$ & 1999 & $6.91(1.82)$ & 0.046 \\
\hline
\end{tabular}

2541 women). Of these individuals, 444 had screendetected T2D, while 638 individuals did not participate in the OGTT at the baseline and were excluded from this study. Thus 2798 individuals participated in this study. ${ }^{15}$ There were altogether $484(17.3 \%)$ statin users at baseline among them. Twenty-six of them $(5.4 \%)$ had started statin use no later than 270 days after the first visit.

\section{Statistical analyses}

Statistical analyses were performed by using SAS (V.9.2) for Windows and they were based on percentage distributions and $\chi^{2}$ test and independent sample $\mathrm{t}$ test. Analysis of covariance (ANCOVA) was used to study the differences between the statin users and non-users in changes of fasting glucose values during the follow-up after adjusting for age, sex, weight, CVD disease, use of antihypertensive and/or coronary artery disease medication and fasting glucose at baseline and 1-year weight change as a continuous variable. Furthermore, separate logistic regression analyses were conducted for statin users and non-users to study the effect of weight loss $(\leq 0.8$ or $>0.8 \mathrm{~kg})$ on T2D risk after adjusting for age, sex, weight, CVD diseases, use of above-mentioned medication and glucose tolerance and at the baseline.

\section{RESULTS}

There were more men than women using statins at baseline $(23 \%$ vs $14.5 \%, \mathrm{p}<0.001)$. Statin users were older than non-users (table 1 ). In addition, statin users had lower BMI, diastolic BP, total cholesterol, high-density lipoprotein (HDL) cholesterol and low-density lipoprotein (LDL) cholesterol than non-users at the baseline,

Table 2 One-year changes in cardio-metabolic risk factors according to use of statins at the baseline in a high-risk cohort of the FIN-D2D

\begin{tabular}{|c|c|c|c|c|c|}
\hline \multirow[b]{3}{*}{ Variable } & \multicolumn{4}{|c|}{ Use of statins } & \multirow[b]{3}{*}{ p Value } \\
\hline & \multicolumn{2}{|c|}{ User } & \multicolumn{2}{|c|}{ Non-user } & \\
\hline & $\mathbf{N}$ & Mean (SD) & $\mathbf{N}$ & Mean (SD) & \\
\hline Weight (kg) & 484 & $-0.80(4.17)$ & 2314 & $-1.2(5.88)$ & 0.143 \\
\hline BMI $\left(\mathrm{kg} / \mathrm{m}^{2}\right)$ & 484 & $-0.29(1.48)$ & 2302 & $-0.45(2.03)$ & 0.111 \\
\hline Systolic BP (mm Hg) & 472 & $-1.9(15.1)$ & 2276 & $-1.5(14.8)$ & 0.531 \\
\hline Diastolic BP (mm Hg) & 472 & $-1.4(8.33)$ & 2276 & $-1.6(8.59)$ & 0.579 \\
\hline Total cholesterol (mmol/l) & 429 & $-0.15(0.91)$ & 2051 & $-0.18(0.80)$ & 0.560 \\
\hline HDL cholesterol (mmol/l) & 425 & $0.03(0.24)$ & 2028 & $0.03(0.29)$ & 0.981 \\
\hline LDL cholesterol (mmol/l) & 415 & $-0.17(0.81)$ & 1979 & $-0.18(0.73)$ & 0.850 \\
\hline Triglycerides $(\mathrm{mmol} / \mathrm{l})$ & 424 & $-0.02(0.77)$ & 2019 & $-0.06(0.84)$ & 0.314 \\
\hline Fasting glucose (mmol/l) & 424 & $0.08(0.60)$ & 2008 & $-0.00(0.57)$ & 0.008 \\
\hline $2 \mathrm{~h}$ glucose $(\mathrm{mmol} / \mathrm{l})$ & 421 & $-0.13(1.74)$ & 1999 & $-0.10(1.70)$ & 0.721 \\
\hline
\end{tabular}


whereas the levels of triglycerides, fasting glucose and $2 \mathrm{~h}$ glucose were higher in statin users compared to non-users (table 1). Bivariate analyses between statin use and changes in cardio-metabolic characteristics showed that the changes in weight, BMI, systolic and diastolic $\mathrm{BP}$, total, HDL and LDL cholesterol, triglycerides and $2 \mathrm{~h}$ glucose were similar in statin users and non-users, the only exception being in the change of fasting glucose. Fasting glucose level increased by $0.08 \mathrm{mmol} / 1$ during the follow-up in statin users but remained unchanged in non-users (table 2), the difference being $0.074 \mathrm{mmol} / 1$ (95\% CI 0.014 to 0.134 ) between the groups. The difference was significant even after adjustment for age, sex and fasting glucose at the baseline $(p=0.006)$ and after adding weight and CVD at the baseline $(p=0.015)$, and even when weight change $(p=0.026)$ and antihypertensive and/or coronary artery disease medication were added to the model $(\mathrm{p}=0.042)$.

Figure 1 presents the changes in fasting and $2 \mathrm{~h}$ glucose in statin users and non-users according to weight loss. In individuals whose weight loss was $\leq 0.8 \mathrm{~kg}$, fasting glucose increased more in statin users compared to non-users $(\mathrm{p}=0.055$ after adjustment for age, sex, weight, CVD, medication and fasting glucose at the baseline). In those with weight loss of $>0.8 \mathrm{~kg}$, the reduction in fasting glucose was not statistically significantly different between statin users and non-users $(p=0.330$ after adjustment, figure 1 ).

\section{Statin therapy and incidence of T2D}

A total of $7.5 \%(n=31)$ of the individuals who were statin users developed T2D during the follow-up compared to $6.5 \%(\mathrm{n}=126)$ of non-users $(\mathrm{p}=0.442)$. OR was 1.17 (95\% CI 0.78 to 1.76 ) for users compared to non-users. Logistic regression analyses showed that only in statin non-users, weight loss was associated statistically significantly with the reduction of T2D even after adjustment for age, sex, weight, CVD, use of antihypertensive and or coronary artery disease medication and glucose tolerance status at the baseline (table 3). However, the corresponding interaction test for statin users vs non-users was not statistically significant, suggesting that the association was not different in these two groups. In both statin users and non-users, IGT at baseline predicted a very high risk of developing T2D (table 3).

\section{DISCUSSION}

Our results showed that the incidence of T2D during the 1-year follow-up did not differ between statin users and non-users. Interestingly, however, use of statins was related to increase in fasting glucose, and the change in fasting glucose was significantly different between these two groups even after adjustment for age, sex, weight, CVD disease, fasting glucose and use of antihypertensive and/or coronary artery disease medication at baseline and weight change.
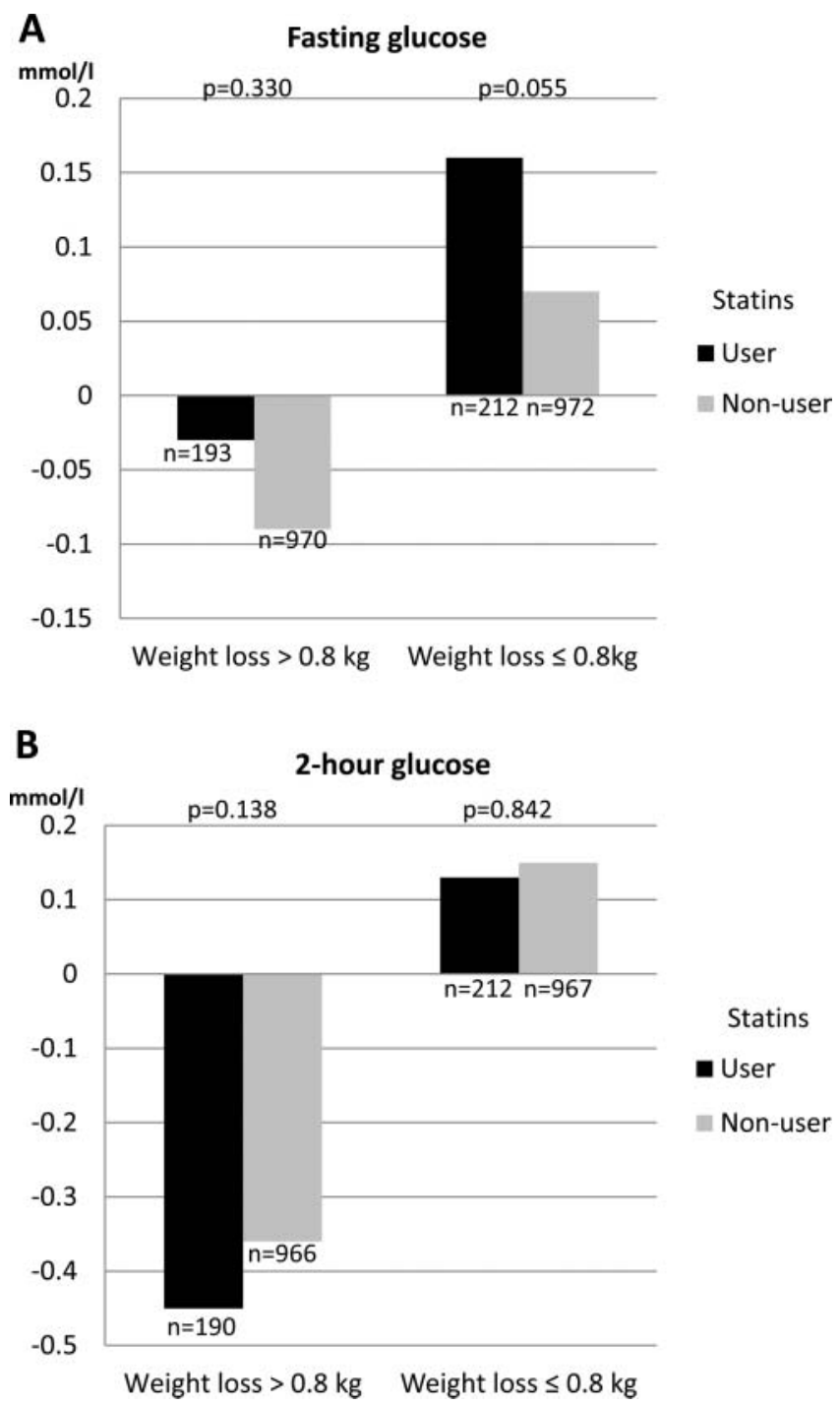

Figure 1 Change in glucose metabolism. Change in fasting glucose $(\mathrm{mmol} / \mathrm{l})$ and $2 \mathrm{~h}$ glucose $(\mathrm{mmol} / \mathrm{l})$ during the 1-year follow-up in statin users and non-users according to weight loss. $p$ Values adjusted for age, sex, weight, cardiovascular diseases (CVD), use of antihypertensive/coronary artery disease medication and baseline value of each outcome variable.

As far as we know, this is the first study examining the association of lifestyle intervention on the risk of T2D according to the use of statins. This question is of utmost clinical importance since we now know that T2D is preventable by lifestyle changes. ${ }^{10-14} \mathrm{~A}$ slight increase in fasting glucose during the follow-up in statin users in our study is in line with the findings from a prospective follow-up study comprising both diabetic and nondiabetic individuals without any lifestyle intervention. ${ }^{24}$ In addition, another study also showed slight increase in fasting glucose in diabetic patients. ${ }^{25}$ In our study fasting glucose increased by $0.08 \mathrm{mmol} / \mathrm{l}$ in statin users, whereas there was a slight decrease in $2 \mathrm{~h}$ glucose values in both groups after 1-year of follow-up. An increase in fasting 
Table 3 Logistic regression analyses concerning T2D risk according to 1-year weight change for both statin users and non-users groups

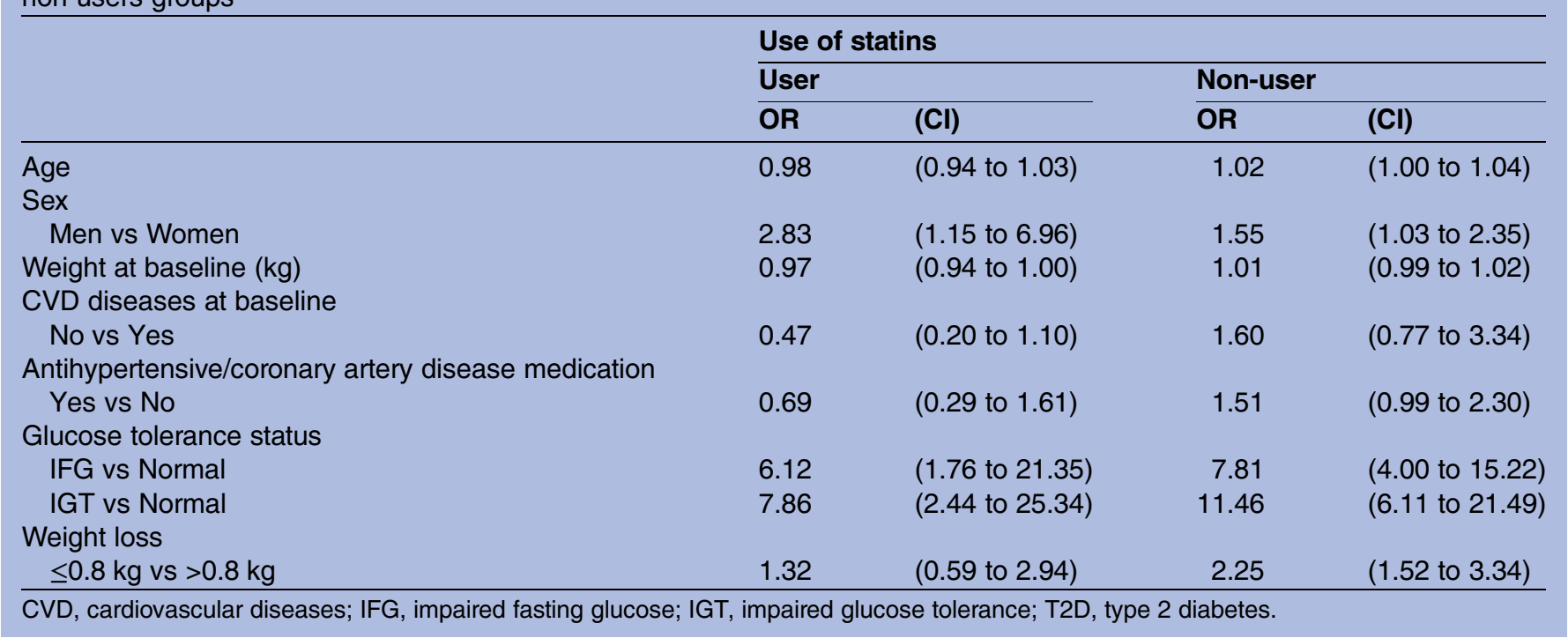

glucose in statin users suggests deterioration in insulin secretion capacity in statin users. ${ }^{26}$ Besides its putative effect on insulin secretion statins may also interfere with insulin sensitivity. ${ }^{27}$ Nevertheless, in this study, $2 \mathrm{~h}$ glucose values, reflecting insulin sensitivity, were similarly decreased in statin users and non-users. It is not known whether statins have any acute effects on fasting glucose level, and we do not know whether people in our study had taken statin tablets in the morning or evening. From the clinical point of view, we want to emphasise even more intensive lifestyle interventions, ${ }^{14}{ }^{28}$ for high-risk patients using statins in order to prevent T2D. We also highlight that the role of lifestyle in the prevention of T2D in statin users should be investigated in larger intervention studies with longer follow-up.

The strength of our study is that glucose tolerance status was based on the OGTT, ${ }^{21}$ and that IGT, that is, post-challenge hyperglycaemia, was strongly related to the development of T2D. Note that this information is missing from all previous studies that have investigated the relation between statins and T2D risk. In addition, the advantage of our study was that we were able to examine the changes in various cardio-metabolic risk factors according to the use of statins. The main shortcomings were that a high number of individuals who were screened at baseline did not participate in the intervention programme, ${ }^{15}$ and thus the present results can be generalised only to a subgroup of high-risk individuals who also may present highly motivated individuals. Furthermore, the number of statin users was quite small and the use of statins was self-reported and not based on health records, which is why we did not have detailed information on the agents and doses used. We are also unable to say how long these individuals had used statins. We also conducted analyses on the use of statins and their association to changes in glucose metabolism and T2D risk excluding those 26 individuals who started to use statins no later than 270 days after the first visit. These results were very similar (data not shown) to findings presented here. Due to the nature of our data we cannot elicit a causal relationship. In addition, our study is likely to be underpowered to demonstrate any statistical difference in T2D risk according to the use of statins due to the short follow-up period. There may also be other factors (eg, variations in how the interventions were implemented in the centres) that may have an effect on the associations of statins and fasting glucose which we were not able to take into account in our study.

In summary, statins may have unfavourable effects on glucose metabolism and hamper the beneficial effects of lifestyle intervention in people at high risk of T2D, but the benefits of statin therapy for the prevention of CVD in people with an increased risk are still unequivocal.

\section{Author affiliations}

${ }^{1}$ Pirkanmaa Hospital District, Tampere, Finland

${ }^{2}$ Institute of Health Sciences, University of Oulu, Oulu, Finland

${ }^{3}$ Unit of General Practice, Oulu University Hospital, Oulu, Finland

${ }^{4}$ Finnish Diabetes Association, Tampere, Finland

${ }^{5}$ Diabetes Prevention Unit, National Institute for Health and Welfare, Helsinki, Finland

${ }^{6}$ Unit of Family Practice, Central Finland Health Care District, Jyväskylä, Finland

${ }^{7}$ Department of Internal Medicine, Seinäjoki Central Hospital, Seinäjoki, Finland

${ }^{8}$ Department of Medicine, Kuopio University Hospital, Kuopio, Finland ${ }^{9}$ Center for Vascular Prevention, Danube-University Krems, Krems, Austria

${ }^{10}$ Institute of Public Health and Clinical Nutrition, University of Eastern Finland, Kuopio, Finland

${ }^{11}$ Research Unit, Kuopio University Hospital, Kuopio, Finland

Contributors NR and MU designed this post hoc substudy, wrote the manuscript and NR is the guarantor. $\mathrm{JJ}$ analysed the data. All authors contributed to the collection and interpretation of data, and the writing process of the manuscript. All authors declare that they accept full 
responsibility for the conduct of the study, had access to the data and controlled the decision to publish.

\section{Competing interests None.}

Funding FIN-D2D was supported by financing from the hospital districts of Pirkanmaa, Southern Ostrobothnia, North Ostrobothnia, Central Finland and Northern Savo, the Finnish National Public Health Institute, the Finnish Diabetes Association, the Ministry of Social Affairs and Health in Finland, Finland's Slottery Machine Association, the Academy of Finland (grant number 129293) and Commission of the European Communities, Directorate C-Public Health (grant agreement no. 2004310) in cooperation with the FIN-D2D Study Group, and the Steering Committee: Huttunen J, Kesäniemi A Kiuru S, Niskanen L, Oksa H, Pihlajamäki J, Puolakka J, Puska P, Saaristo T, Vanhala $\mathrm{M}$ and Uusitupa $\mathrm{M}$. The funders have no role (except for the hospital districts) in study design, data collection and analysis, decision to publish or preparation of the manuscript.

Ethics approval Ministry of Social Affairs and Health in Finland gave the permission to collect data from healthcare units specifically for scientific evaluation purposes to National Public Health Institute.

Provenance and peer review Not commissioned; externally peer reviewed.

Data sharing statement No additional data are available.

\section{REFERENCES}

1. Baigent C, Keech A, Kearney PM, et al. Efficacy and safety of cholesterol-lowering treatment: prospective meta-analysis of data from 90,056 participants in 14 randomised trials of statins. Lancet 2005;366:1267-78.

2. Baigent $\mathrm{C}$, Blackwell L, Emberson $\mathrm{J}$, et al. Efficacy and safety of more intensive lowering of LDL cholesterol: a meta-analysis of data from 170,000 participants in 26 randomised trials. Lancet 2010;376:1670-81.

3. Kearney PM, Blackwell L, Collins R, et al. Efficacy of cholesterol-lowering therapy in 18,686 people with diabetes in 14 randomised trials of statins: a meta-analysis. Lancet 2008;371:117-25

4. Rajpathak SN, Kumbhani DJ, Crandall J, et al. Statin therapy and risk of developing type 2 diabetes: a meta-analysis. Diabetes Care 2009;32:1924-9.

5. Ridker PM, Danielson E, Fonseca FA, et al. Rosuvastatin to prevent vascular events in men and women with elevated C-reactive protein. N Engl J Med 2008;359:2195-207.

6. Waters DD, Ho JE, DeMicco DA, et al. Predictors of new-onse diabetes in patients treated with atorvastatin: results from 3 large randomized clinical trials. J Am Coll Cardiol 2011;57:1535-45.

7. Sattar N, Preiss D, Murray HM, et al. Statins and risk of incident diabetes: a collaborative meta-analysis of randomised statin trials. Lancet 2010;375:735-42.

8. Freeman DJ, Norrie J, Sattar N, et al. Pravastatin and the development of diabetes mellitus: evidence for a protective treatment effect in the West of Scotland Coronary Prevention Study. Circulation 2001;103:357-62.

9. Preiss D, Seshasai SR, Welsh $\mathrm{P}$, et al. Risk of incident diabetes with intensive-dose compared with moderate-dose statin therapy: a meta-analysis. JAMA 2011;305:2556-64.

10. Knowler WC, Barrett-Connor E, Fowler SE, et al. Reduction in the incidence of type 2 diabetes with lifestyle intervention or metformin. N Engl J Med 2002;346:393-403.
11. Kosaka K, Noda M, Kuzuya T. Prevention of type 2 diabetes by lifestyle intervention: a Japanese trial in IGT males. Diabetes Res Clin Pract 2005;67:152-62.

12. Pan XR, Li GW, Hu YH, et al. Effects of diet and exercise in preventing NIDDM in people with impaired glucose tolerance. The Da Qing IGT and diabetes study. Diabetes Care 1997;20:537-44.

13. Ramachandran A, Snehalatha C, Mary S, et al. The Indian Diabetes Prevention Programme shows that lifestyle modification and metformin prevent type 2 diabetes in Asian Indian subjects with impaired glucose tolerance (IDPP-1). Diabetologia 2006;49:289-97.

14. Tuomilehto J, Lindström J, Eriksson JG, et al. Prevention of type 2 diabetes mellitus by changes in lifestyle among subjects with impaired glucose tolerance. N Engl J Med 2001;344:1343-50.

15. Saaristo T, Moilanen L, Korpi-Hyövälti E, et al. Lifestyle intervention for prevention of type 2 diabetes in primary health care: one-year follow-up of the Finnish National Diabetes Prevention Program (FIN-D2D). Diabetes Care 2010;33:2146-51.

16. Saaristo T, Peltonen M, Keinänen-Kiukaanniemi S, et al. National type 2 diabetes prevention programme in Finland: FIN-D2D. Int $J$ Circumpolar Health 2007;66:101-12.

17. The Finnish Diabetes Association.Implementation of Type 2 Diabetes Prevention Plan: Project Plan 2003-2007, FIN-D2D Project.[Article online]. 2006. http://www.diabetes.fi/d-kauppa/dehko/ dehko_in_english/implementation_of_type_2_diabetes_prevention. project plan 2003-2007.657.shtml (accessed 3 May 2012).

18. Lindström J, Tuomilehto J. The diabetes risk score: a practical tool to predict type 2 diabetes risk. Diabetes Care 2003;26:725-31.

19. Saaristo T, Moilanen L, Jokelainen J, et al. Cardiometabolic profile of people screened for high risk of type 2 diabetes in a national diabetes prevention programme (FIN-D2D). Prim Care Diabetes 2010;4:231-9.

20. Eriksson J, Lindström J, Valle T, et al. Prevention of type II diabetes in subjects with impaired glucose tolerance: the Diabetes Prevention Study (DPS) in Finland. Study design and 1-year interim report on the feasibility of the lifestyle intervention programme. Diabetologia 1999;42:793-801.

21. World Health Organization. Definition, diagnosis and classification of diabetes mellitus and its complications. Report of a WHO consultation. Part 1: Diagnosis and classification of diabetes mellitus. Geneva, Switzerland: World Health Organization, 1999. Report No 99.2

22. Working group set up by the Finnish Medical Society Duodecim and Finnish Society of Internal Medicine. Dyslipidemias. 2009. http:// www.kaypahoito.fi/web/kh/suositukset/naytaartikkeli/tunnus/ ccs00055?hakusana=dyslipidaemias (accessed 3 May 2012)

23. KELA. Drug treatment statistics of the most common diseases entitling the patients to reimbursement under the Special Refund Categories, in 2010. 29.6.2011. 2011. http://www.kela.fi/in/internet/ liite.nsf/NET/280611170238PB/\$File/eritlaak.pdf?OpenElement. (accessed 3 May 2012)

24. Sukhija R, Prayaga S, Marashdeh M, et al. Effect of statins on fasting plasma glucose in diabetic and nondiabetic patients. J Investig Med 2009;57:495-9.

25. Simsek S, Schalkwijk CG, Wolffenbuttel BH. Effects of rosuvastatin and atorvastatin on glycaemic control in type 2 diabetes-the CORALL study. Diabet Med 2012;29:628-31.

26. Sampson UK, Linton MF, Fazio S. Are statins diabetogenic? Curr Opin Cardiol 2011;26:342-7.

27. Baker WL, Talati R, White CM, et al. Differing effect of statins on insulin sensitivity in non-diabetics: a systematic review and meta-analysis. Diabetes Res Clin Pract 2010;87:98-107.

28. Jula A, Marniemi J, Huupponen R, et al. Effects of diet and simvastatin on serum lipids, insulin and antioxidants in hypercholesterolemic men. A randomized controlled trial. JAMA 2002;287:598-605 